CARTA AL EDITOR

\section{DESIGUALDADES SOCIALES EN LA MORTALIDAD DURANTE LA COVID-19 EN LIMA Y CALLAO}

\section{SOCIAL INEQUALITIES IN MORTALITY DURING COVID-19 IN LIMA AND CALLAO}

\author{
Oscar J. Mújica(1) 1,a,b, Paul E. Pachas (10,b \\ ${ }^{1}$ Departamento de Evidencia e Inteligencia para la Acción en Salud \\ Organización Panamericana de la Salud/Organización Mundial de la \\ Salud, Washington DC, EE. UU. \\ ${ }^{2}$ Centro Nacional de Salud Pública, Instituto Nacional de Salud, Lima, Perú. \\ a Médico cirujano, magister en salud pública; ${ }^{\text {b }}$ Médico cirujano, especialista \\ en epidemiología de campo.
}

Sr. Editor: Hasta el cinco de noviembre de 2020, el Ministerio de Salud del Perú había confirmado la ocurrencia de 34730 defunciones por la COVID-19, de las cuales 40,7\% se concentraba en Lima Metropolitana, el epicentro de la pandemia en el país ${ }^{(1)}$. Asimismo, el Sistema Informático Nacional de Defunciones (SINADEF) registró 79544 defunciones entre el 1 de enero y el 31 de octubre de 2020 en los 50 distritos de la provincia de Lima y el Callao; en contraste, para el mismo periodo y ámbito geográfico, se había registrado 29615 defunciones en el año 2019, 27956 en 2018 y 24823 en $2017^{(2)}$. Como se viene reportando en todo el mundo, el exceso de mortalidad observado el 2020, en gran medida es atribuible al impacto, directo e indirecto, de la pandemia de la COVID-19 ${ }^{(3)}$.

Utilizando los datos del SINADEF para los 50 distritos de Lima y Callao hasta la semana epidemiológica 44 (31 de octubre de 2020), realizamos un análisis exploratorio de la desigualdad social en la mortalidad general y sus tendencias entre los quintiles extremos de distritos según la prevalencia de pobreza monetaria estimada por el Instituto Nacional de Estadística e Informática (INEI) para 2018. Los quintiles extremos se definieron calculando las bisagras de Tukey para los percentiles $0,80(19,2 \%)$ y $0,20(1,9 \%)$ de la distribución ordenada de prevalencia distrital de pobreza monetaria. El quintil distrital en mayor pobreza monetaria (promedio ponderado: 22,1\%) quedó conformado por Ancón, Independencia, Mi Perú, Pachacamac, Pucusana, Puente Piedra, Punta Hermosa, Santa Rosa, Ventanilla y Villa El Salvador y el quintil de menor pobreza monetaria (promedio ponderado: 1,0\%) por Jesús María, La Molina, Lince, Magdalena

\footnotetext{
Citar como: Mújica OJ, Pachas PE. Desigualdades sociales en la mortalidad durante la COVID-19 en Lima y Callao. Rev Peru Med Exp Salud Publica. 2021;38(1):183-4. doi: https://doi.org/10.17843/rpmesp.2021.381.6740.

Correspondencia: Oscar J Mújica; mujicaos@paho.org
}

Recibido: 11/11/2020 Aprobado: 02/12/2020 En línea: 27/01/2021 del Mar, Miraflores, Pueblo Libre, San Borja, San Isidro, San Miguel y Santiago de Surco. Para cada distrito y por cada quintil extremo se estimaron las tasas de mortalidad general ajustadas por edad, usando la población estándar 2000-2025 de la Organización Mundial de la Salud. Además, se calculó para cada distrito el porcentaje de fallecidos con nivel de instrucción inferior a secundaria completa como estratificador social y el índice de Kuznets absoluto y relativo para cuantificar las brechas de desigualdad ${ }^{(4)}$.

Nuestros hallazgos muestran una marcada desigualdad en la mortalidad general entre los quintiles extremos de pobreza monetaria distritales de las provincias de Lima y Callao, evidente después del confinamiento domiciliario y durante el periodo de mayor actividad pandémica (Figura 1). Aunque la mortalidad se elevó en ambos quintiles, el incremento fue notoriamente mayor en el quintil de distritos de mayor pobreza; de hecho, durante el pico epidémico observado en la semana 23 ( 31 de mayo a 6 de junio), la tasa de mortalidad en el quintil más pobre (42,0 x 100000$)$ fue 2,2 veces (brecha relativa de Kuznets) la tasa en el quintil menos pobre (19,6 x 100 000), representando un exceso de mortalidad de 22,4 defunciones por 100000 habitantes de los distritos en mayor pobreza monetaria (brecha absoluta de Kuznets).

Se confirmó también la notable desigualdad educacional entre ambos quintiles distritales: el porcentaje de fallecidos con nivel de instrucción menor a secundaria completa fue substantivamente más alto en el quintil de distritos más pobres $(52,2 \%)$ que en el quintil de distritos menos pobres $(18,7 \%)(\mathrm{p}<0,0001)$. Sin embargo, la tendencia de la mortalidad proporcional por nivel de instrucción se ha mantenido constante durante el periodo estudiado (Material suplementario), lo cual sugiere que la brecha de desigualdad en el riesgo de morir observada en 2020 quedó determinada por la desigualdad económica. En términos generales, alrededor de $75 \%$ del exceso de mortalidad entre los quintiles distritales extremos de pobreza monetaria de Lima y Callao en lo que va del 2020 se ha concentrado desproporcionadamente en el quintil de mayor pobreza monetaria.

Siendo un análisis exploratorio de datos socialmente agregados a nivel ecológico, existe la limitación de no establecer relaciones causales, aunque satisface el objetivo de extraer patrones de los datos, proporcionando evidencia para informar decisiones de política sanitaria y especialmente la necesidad de establecer medidas de protección social diferenciadas y progresivas para mitigar el impacto en el riesgo de morir. La inherente comparación dicotómica impuesta por el análisis de brechas de desigualdad, que se limita a contrastar grupos extremos socialmente determinados, debe ser confirmada con estudios de gradientes de desigualdad, a lo largo de toda la jerarquía social a partir de microdatos. Un detalle adicional a considerar es el alto porcentaje de valores ausentes sobre nivel de instrucción en la base de datos del SINADEF, $39,8 \%$ en el quintil más pobre y $31,3 \%$ en el quintil 


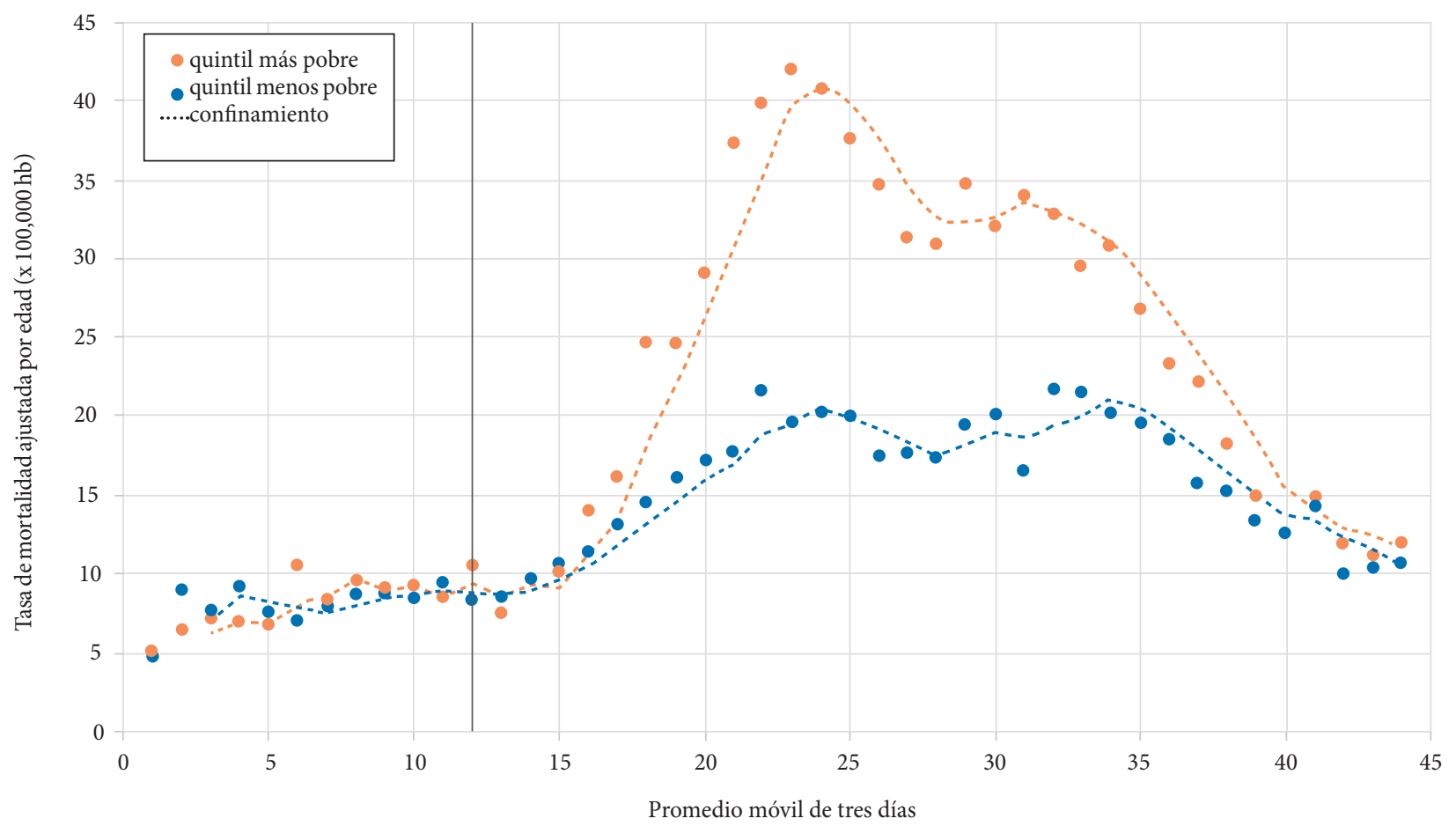

Figura 1. Tendencias de la mortalidad general por semanas epidemiológicas (1 de enero al 31 de octubre del 2020), en los quintiles de distritos con mayor y menor pobreza monetaria en Lima y el Callao.

menos pobre. No obstante, la correspondencia entre deprivación económica y deprivación educacional con el exceso de mortalidad observado queda evidenciada.

La marcada desigualdad social en el Perú y el resto del continente americano, aunada a la falta de acceso universal a la salud, determina la profunda desigualdad en salud y en sus resultados de más alto costo social, como es la mortalidad ${ }^{(5)}$. En el escenario pandémico actual, se hace necesario diseñar políticas públicas y poner en práctica estrategias e intervenciones sensibles a la equidad, que restituyan el principio de justicia social y brinden igualdad de oportunidades para mitigar la pandemia de la COVID-19 y sus consecuencias de corto, mediano y largo plazos.

Contribuciones de los autores: OJM y PEP participaron en la concepción del artículo, la recolección de datos, la interpretación de datos, la revisión crítica del artículo y la aprobación de la versión final. OJM además realizó el análisis cuantitativo de datos y la redacción del artículo.

Financiamiento: Autofinanciado.

Conflictos de interés: Los autores declaran no tener conflictos de interés.

Declaración: Las opiniones expresadas en este artículo son únicamente responsabilidad de los autores y no reflejan necesariamente los criterios ni la política de la Organización Panamericana de la Salud.
Material suplementario: Disponible en la versión electrónica de la RPMESP.

\section{REFERENCIAS BIBLIOGRÁFICAS}

1. Gobierno del Perú. Ministerio de Salud. Situación Actual COVID-19, 5 de noviembre 2020. Centro Nacional de Epidemiología, Prevención y Control de Enfermedades. Disponible en: https://www.dge.gob.pe/portal/ docs/tools/coronavirus/coronavirus051120.pdf.

2. Gobierno del Perú. Plataforma Nacional de Datos Abiertos. Dataset de información de fallecidos del Sistema Informático Nacional de Defunciones [Ministerio de Salud]. [Fecha de acceso: 6 de noviembre de 2020]. Disponible en: https://www.datosabiertos.gob.pe/dataset/informaci\%C3\%B3n-de-fallecidos-del-sistema-inform\%C3\%A1tico-nacional-de-defunciones-sinadef-ministerio.

3. Vestergaard LS, Nielsen J, Richter L, Schmid D, Bustos N, Braeye T, et al. Excess all-cause mortality during the COVID-19 pandemic in Europe-preliminary pooled estimates from the EuroMOMO network, March to April 2020. Euro Surveill. 2020;25(26):2001214. doi: 10.2807/1560-7917. ES.2020.25.26.2001214.

4. Organización Panamericana de la Salud. Manual para el Monitoreo de las Desigualdades en Salud, con especial énfasis en países de ingresos medianos y bajos. Washington DC: Organización Panamericana de la Salud; 2016. [Fecha de acceso: 6 de noviembre de 2020]. Disponible en: https://www.paho.org/hq/index.php?option=com_docman\&task=doc_download\&gid=36348\&Itemid=270\&lang=e.

5. Organización Panamericana de la Salud. Salud en las Américas, Edición 2017. Resumen: Panorama Regional y Perfiles de País. Washington DC: OPS; 2017. [Fecha de acceso: 6 de noviembre de 2020]. Disponible en: https://www.paho.org/salud-en-las-americas-2017/wp-content/ uploads/2017/09/Print-Version-Spanish.pdf. 